\title{
Dysregulation of ghrelin in diabetes impairs the vascular reparative response to hindlimb ischemia in a mouse model; clinical relevance to peripheral artery disease
}

Joshua Neale

University of Otago

James Pearson

Kokuritsu Junkankibyo Kenkyu Center

Kate Thomas

University of Otago

Hirotsugu Tsuchimouchi

Kokuritsu Junkankibyo Kenkyu Center

Hiroshi Hosoda

Kokuritsu Junkankibyo Kenkyu Center

Masayasu Kojima

Kurume Daigaku

Takahiro Sato

Kurume Daigaku

Gregory Jones

University of Otago

Adam Denny

University of Otago

Lorna Daniels

University of Otago

Dhananjie Chandrasekera

University of Otago

Ping Liu

University of Otago

Andre van Rij

University of Otago

Rajesh Katare

University of Otago

Daryl Schwenke ( $\square$ daryl.schwenke@otago.ac.nz )

University of Otago https://orcid.org/0000-0002-4506-2948 
Original investigation

Keywords: Ghrelin, peripheral artery disease, diabetes, microRNA, angiogenesis

Posted Date: April 15th, 2020

DOI: https://doi.org/10.21203/rs.3.rs-21827/v1

License: (c) (1) This work is licensed under a Creative Commons Attribution 4.0 International License. Read Full License

Version of Record: A version of this preprint was published at Scientific Reports on August 12th, 2020. See the published version at https://doi.org/10.1038/s41598-020-70391-6. 


\section{Abstract}

Background: Type 2 diabetes is a prominent risk factor for peripheral artery disease (PAD). Yet, the mechanistic link between diabetes and PAD remains unclear. This study proposes that dysregulation of the endogenous hormone ghrelin, a potent modulator of vascular function, underpins the causal link between diabetes and PAD. Moreover, this study aimed to demonstrate the therapeutic potential of exogenous ghrelin in a diabetic mouse model of PAD.

Methods: Standard ELISA analysis was used to quantify and compare circulating levels of ghrelin between i) human diabetic patients with or without PAD (clinic) and ii) $\mathrm{db} / \mathrm{db}$ diabetic and non-diabetic mice (lab). Db/db mice underwent unilateral hindlimb ischaemia (HLI) for 14 days and treated with or without exogenous ghrelin ( $150 \mu \mathrm{g} / \mathrm{kg} /$ day.) Subsequently vascular reparation, angiogenesis, hindlimb perfusion, structure and function were assessed using laser Doppler imaging, micro-CT, microangiography, and protein and micro-RNA (miRNA) analysis. We further examined hindlimb perfusion recovery of ghrelin $\mathrm{KO}$ mice to determine whether an impaired vascular response to $\mathrm{HLI}$ is linked to ghrelin dysregulation in diabetes.

Results: Patients with PAD, with or without diabetes, had significantly lower circulating levels of endogenous ghrelin, compared to healthy individuals. Diabetic $\mathrm{db} / \mathrm{db}$ mice had ghrelin levels that were only $7 \%$ of non-diabetic mice. The vascular reparative capacity of diabetic $\mathrm{db} / \mathrm{db}$ mice in response to $\mathrm{HLI}$ was impaired compared to non-diabetic mice and, importantly, comparable to ghrelin KO mice. Daily therapeutic treatment of $\mathrm{db} / \mathrm{db}$ mice with ghrelin for 14 days post $\mathrm{HLI}$, stimulated angiogenesis, and improved skeletal muscle architecture and cell survival, which was associated with an increase in proangiogenic miRNAs-126 and -132 .

Conclusions: These findings unmask an important role for endogenous ghrelin in vascular repair following limb ischemia, which appears to be downregulated in diabetic patients. Moreover, these results implicate exogenous ghrelin as a potential novel therapy to enhance perfusion in patients with lower limb PAD, especially in diabetics.

\section{Background}

Type 2 diabetes mellitus (T2DM) is now a global pandemic that shows no sign of abatement. Diabetic patients have a greater predisposition to cardiovascular disease, in particular, T2DM is one of the most significant risk factors for lower limb peripheral artery disease (PAD). PAD is the progressive narrowing or occlusion of arteries as a result of atherosclerosis ${ }^{1}$, which effectively increases the risk of mortality ${ }^{2}$. In 2015, it was estimated that over 236 million adults worldwide were living with PAD ${ }^{3}$. Importantly, PAD is one of the most common cardiovascular manifestations seen in T2DM patients ${ }^{4}$. A primary treatment for patients with severe PAD is surgical and endovascular revascularization ${ }^{2}$. However, despite considerable advances in surgical treatment, not all diabetic PAD patients are suited for surgical interventions ${ }^{5}$. In the 
clinic, 'therapeutic angiogenesis' remains a major challenge, as the use of pro-angiogenic agents or gene therapy have failed to translate to routine use in patients with PAD or diabetic PAD.

Recent studies indicate that the peptide hormone ghrelin may have a significant angiogenic role in PAD ${ }^{6}$. Ghrelin, first discovered in $1999^{7}$, circulates in two distinct forms: acylated ghrelin (AG) and des-acylated ghrelin (DAG). Exogenous ghrelin has been shown to have an angiogenic potential in vitro ${ }^{8,9}$ and in vivo $^{10-12}$, whilst also protecting against apoptosis ${ }^{13}$ and inflammation ${ }^{14}$, all of which are highly desirable mechanisms for the treatment of PAD. This is particularly important for patients with T2DM since decreased circulating levels of ghrelin is significantly associated with abdominal adiposity and insulin resistance ${ }^{15}$.

We have previously shown that exogenous AG induces functional therapeutic angiogenesis in a young non-diabetic murine model of PAD, which was associated with the activation of key pro-angiogenic microRNAs (miRNAs) ${ }^{11}$. miRNAs are endogenous, small, non-coding ribonucleic acids of $\sim 20-22$ nucleotides which regulate gene expression at the post-transcriptional level, by either translational suppression or by mRNA degradation ${ }^{16}$.

Here, we first aimed to conduct a small clinical study to investigate whether endogenous ghrelin is dysregulated in humans with PAD, with or without accompanying T2DM. Subsequently, we aimed to determine whether the vascular reparative response to hindlimb ischemia is impaired in diabetic $\mathrm{db} / \mathrm{db}$ mice and, elucidate the role of endogenous ghrelin in driving the vascular reparative response to ischemia using ghrelin knock out mice. Finally, we aimed to ascertain the therapeutic potential of exogenous ghrelin for promoting functional angiogenesis in an aged, T2DM murine model of PAD.

\section{Materials And Methods}

A detailed description of the methods is provided in the online 'Supplementary Methods' file.

\section{Clinical study - plasma ghrelin in human PAD patients with or without diabetes}

Four cohorts of patients were studied: 1) PAD patients with T2DM (PAD+T2DM), 2) PAD patients without T2DM (PAD), 3) T2DM patients with no PAD (T2DM), and 4) control subjects with no T2DM or PAD (Control). Ethical approval was obtained from the University of Otago Human Ethics Committee (Health) (H17/012), and the study conformed to the standards set by the Declaration of Helsinki. All participants were 50 years of age or older, and all provided written consent. Participant demographics are presented in Table 1.

A venous blood sample was drawn, centrifuged, snap-frozen and stored at $-80^{\circ} \mathrm{C}$. Human acylated ghrelin (AG) and des-acylated ghrelin (DAG) were quantified as previously described ${ }^{17}$ using the easy sampling enzyme immunoassay kits purchased from Bertin Pharma (Montigny-le-Bretonneux, France; Cat No: A05306 and A05319 respectively). 
Experimental study - role of ghrelin in vascular reparation following hindlimb ischemia

\section{Animals}

All animal experimentation was approved by the Animal Ethics Committee of the University of Otago, New Zealand, and the National Cerebral and Cardiovascular Centre, Japan. All procedures are reported in accordance with the ARRIVE guidelines. Diabetic db/db (DM; DM, BKS.CG-DOCK7 ${ }^{M}+/+\mathrm{Lepr}^{\mathrm{db}} / \mathrm{J}$ ) and non-diabetic (ND; $\mathrm{db} /+$ ) littermates were obtained from Jacksons Laboratories, Japan, or bred at the Hercus-Taieri breeding facility at the University of Otago.

Ghrelin knock out mice (KO - knockout of the preproghrelin gene), and their wildtype littermates (WT) were bred at the Hercus-Taieri breeding facility (New Zealand) or the National Cerebral and Cardiovascular Centre (Japan).

\section{Hindlimb Ischemia (HLI)}

Using standard aseptic procedures, unilateral limb ischemia of the upper left femoral artery was induced by dual ligation of the femoral artery (with a 6-0 silk suture) at two distinct points as well as electrocoagulation as previously described ${ }^{11}$.

\section{Laser Doppler Imaging}

Hindlimb blood flow was sequentially analysed 1 hour and then again at 3, 7, 10, and 14 days postsurgery in the ischemic and contralateral feet. Hindlimb flow was measured using a real-time microcirculation imager (PeriCam PSI HR system, Perimed, Sweden), which operates on the Laser Speckle Contrast Analysis Technology (LASCA) and measures blood perfusion in arbitrary Perfusion Units (PU).

\section{Microangiography}

Vascular function and hindlimb perfusion were assessed and quantified using Synchrotron microangiography as previously described ${ }^{22}$.

\section{Protocol}

Mice were positioned supine above the X-ray source, and a single bolus of contrast agent was injected at high speed $(0.1 \mathrm{ml} @ 0.4 \mathrm{ml} / \mathrm{s})$ into the iliac artery for imaging of left hindlimb vessels. Following baseline imaging, hindlimb angiograms were recorded in response to: i) acetylcholine (ACh, $10 \mu \mathrm{g} / \mathrm{kg} / \mathrm{min}$ for $5 \mathrm{~min}, \mathrm{i} . \mathrm{v}$ ) to assess endothelium-dependent vasodilation, ii) the NO donor sodium nitroprusside (SNP, $5 \mu \mathrm{g} / \mathrm{kg} / \mathrm{min}$ for $5 \mathrm{~min}$, i.v.) to assess endothelium-independent vasodilation and iii) the Rho-Kinase inhibitor Fasudil (20 mg/kg intravenous bolus).

\section{Immunohistochemistry and histological analysis}


For analysis of capillary and arteriole density, gastrocnemius muscle sections $(7 \mu \mathrm{m})$ were stained with isolectin-B4 (Vector Laboratories, B-1205; 1:100) to identify endothelial cells and the smooth muscle marker, a-smooth muscle actin conjugated to Cy3 (Sigma-Aldrich, C6198, 1:100) as previously described ${ }^{20}$. High resolution images were acquired (at x200) and counts from 18 randomly selected fields were averaged and expressed as the number of capillaries and arterioles $(<50 \mu \mathrm{m})$ per $\mathrm{mm}^{2}$ of muscular sections $^{23}$.

DNA fragmentation, associated with apoptosis, was detected in sections $(7 \mathrm{~mm})$ of gastrocnemius muscle using the commercially available terminal deoxynucleotidyl transferase mediated dUTP nick-end labelling (TUNEL) kit (Click-iT Plus TUNEL Alexa Fluor 594 kit, Life Technologies). Sections were counterstained with Isolectin B4 to identify endothelial cells and DAPI to label nuclei.

Skeletal tissue fibrosis was detected in transverse sections $(7 \mu \mathrm{m})$ of gastrocnemius muscle using Mason's Trichrome staining.

\section{RNA isolation and quantitative real-time PCR}

Total RNA was isolated from ischemic gastrocnemius tissue samples using the miRNeasy mini kit (Qiagen). Real-time PCR was performed with Applied Biosystems miRNA assays (miRNAs -126, 132, -206, and -92a) on a StepOnePlus device.

\section{Western blot analyses}

Ischemic gastrocnemius tissue was homogenised and total protein $(20 \mu \mathrm{g})$ was resolved with SDS-PAGE, transferred onto a PVDF membrane, and probed with the following antibodies: VEGFA (SantaCruz Biotechnology, sc-152; 1:1000), BCL-2 (Cell Signaling, 1:1000), AKT (Cell Signaling, 1:1000), and Phospho-AKT ${ }^{\text {ser473 }}$ (Cell Signaling, 1:1000). For detection, goat anti-rabbit secondary (Abcam; 1:2000) conjugated to horseradish peroxidase was used, followed by chemiluminescence reaction with clarity ESC blotting substrate (Bio-Rad).

\section{Mouse ELISA}

Blood $(0.2 \mathrm{ml})$ was collected into a EDTA microvette, stabilised with 4-(2-aminoethyl) benzenesulphonyl fluoride (1:100; A8456 Sigma-Aldrich) ${ }^{17}$, centrifuged (< $1 \mathrm{~min}$ ) at $1500 \mathrm{~g}$, and aliquoted. AG concentrations were quantified using the AG (mouse, rat) easy sampling EIA Kit (Bertin Pharma) according to the manufacturer's instructions.

\section{Volumetric microcomputer tomography and whole-mount imaging}

Hindlimb angioarchitecture was assessed in separate cohorts of mice for each experimental group using microcomputer tomography (micro-CT) analysis. The lower half of the mouse was perfused with Microfil (MV122-Yellow; Flow Tech Inc) scanned on a SkyScan-1172 high-resolution micro-CT system (Skyscan, Aarteselar, Belgium) at a $17.4 \mathrm{~mm}$ resolution, a voltage of $60 \mathrm{kV}$, and a current of $167 \mu \mathrm{A}$. 


\section{Results}

\section{Downregulation of ghrelin in patients with peripheral artery disease and type 2 diabetes}

Circulating levels of ghrelin were analyzed in plasma samples collected from patients with T2DM+PAD, non-diabetic PAD, and their respective controls (non-PAD). Patient characteristics are presented in Table 1. The population comprised equal proportions of male vs female $(P=0.15)$, although age did differ between groups, with PAD and PAD+T2DM participants slightly older than their non-PAD counterparts ( $P$ $<0.002)$. HbA1c and ABI confirmed group allocations with respect to diabetes status and PAD.

Plasma AG in all diseased groups was significantly lower than that of the non-diabetic, non-PAD control subjects ( $P=0.049$, see Supplementary Fig. 1 \& Table 2$)$, although Dunn's correction for multiple comparisons revealed no significant differences in AG between any of the diseased groups. The ROC curves used to determine the threshold value for plasma AG to distinguish diseased groups from controls was $67 \mathrm{pg} / \mathrm{mL}$ (Table 3). Subsequent logistic regression analysis revealed a significant association between $A G<67 \mathrm{pg} / \mathrm{mL}$ and having T2DM, PAD and PAD+T2DM (Table 4).

AG expression has been suggested to decline with age in males ${ }^{27}$. Adjusting the model for age, the significant association remained. Moreover, there was no significant correlation between 'age' and 'plasma $A G$ ' in any of the groups ( $P=0.24$ for all groups). Similarly, there was no significant association between $\mathrm{HbA} 1 \mathrm{c}$ and $\mathrm{AG}$ in any of the groups ( $P=0.15$ for all groups).

\section{Reduced ghrelin levels are associated with impaired vascular reparation following HLI}

To determine if reduced ghrelin levels are associate with impaired recovery of vascular perfusion after $\mathrm{HLI}$, we induced $\mathrm{HLI}$ in aged diabetic $\mathrm{db} / \mathrm{db}$ mice.

\section{Circulating ghrelin levels are reduced in diabetic $\mathrm{db} / \mathrm{db}$ mice}

The plasma AG concentration in $\mathrm{db} / \mathrm{db}$ diabetic mice (DM) prior to HLI (44 $\pm 14 \mathrm{pg} / \mathrm{ml})$ was only $7 \%$ of the non-diabetic (ND) mice (593 $\pm 157 \mathrm{pg} / \mathrm{ml}$; Fig. 1A). Moreover, circulating AG in ND mice progressively increased over 14 days of $\mathrm{HLI}(P<0.01)$, whereas AG did not change following HLI in DM mice.

\section{Hindlimb revascularization and vascular reparation following HLI is impaired in diabetes}

Laser Doppler perfusion imaging revealed that the initial ischemic insult to the hindlimb following HLI was more severe in DM mice compared to ND mice (ischemic to non-ischemic hindlimb perfusion ratio of 0.2 and 0.34 , respectively; Fig. 1B). Although there was a time-dependent restoration of hindlimb blood flow in both ND and DM mice, limb perfusion in DM mice never improved to the same level as seen in the ND mice (Fig. 1B).

At day 14 post-HLI, hindlimb vasculature volume, which comprises the enlarged collateral vessels (microCT; Fig. 1C), as well as capillary and arteriole $(<50 \mu \mathrm{m})$ densities (immunohistochemistry; Fig. 1D) were 
significantly lower in DM mice compared with ND mice.

\section{Specific role of endogenous ghrelin in hindlimb revascularization following HLI}

To selectively identify whether dysregulation of endogenous ghrelin in diabetes underpins the impaired revascularization response following $\mathrm{HLI}$, we quantified the vascular reparative response in ghrelin $\mathrm{KO}$ mice, which lack other confounding effects associated with diabetes.

\section{Ghrelin knockout impairs post-HLI revascularization}

Similar to that previously observed for the diabetic $\mathrm{db} / \mathrm{db}$ mice, ghrelin KO mice had a poorer recovery in hind limb blood flow after 14 days post-HLI (Fig. 2A-B), reduced vascular volume (micro-CT; Fig. 2C-E) and reduced capillary/arteriole density (Fig. 2F-H), compared to the wild type counterpart. Consequently, the functional capacity of the hindlimb was impaired in KO mice (Supplementary Fig. 2).

\section{Microangiographic assessment of vascular function - role of endogenous ghrelin}

Changes in hindlimb vascular function following HLI was assessed based on the vasodilatory responses to ACh (endothelial-dependent) and SNP (endothelial-independent) using microangiography

(Supplementary Fig. 3A-B). In sham mice, the vasodilatory responses of all measured vessels to both ACh and SNP were not significantly different between WT and KO, indicating normal vascular function in KO sham mice (Supplementary Fig. 3C-D). However, following 14 days of HLI, the vasodilatory responses to ACh and SNP, especially the 50-100 $\mu \mathrm{m}$ vessels, were significantly blunted in $\mathrm{KO}$ mice, reflecting impaired vascular function (Supplementary Fig. 3C-D).

The mean arterial blood pressure (MABP) and heart rate (HR) responses to ACh and SNP were mostly similar across all groups (Supplementary Fig. 4).

\section{Ghrelin knockout reduces tissue repair and pro-angiogenic miRNAs following HLI}

HLI was associated with an increase in TUNEL ${ }^{+}$apoptotic cell (Fig. 3A, C) and endothelial cell counts (Fig. 3B, D) in the gastrocnemius muscle, which was significantly higher in KO mice by $154 \%$ and $446 \%$ respectively, compared to WT mice (Fig. 3A, C). In addition, the severity of fibrosis following HLI was amplified in KO mice compared to WT mice (Fig. 3E, F).

Consistent with the observed lack of angiogenesis and arteriogenesis in $\mathrm{KO}$ mice following 14 days of $\mathrm{HLI}$, the expression of pro-angiogenic miRNAs -126 and -132 (Supplementary Fig. 5A-B), as well as the downstream angiogenic protein VEGF (Supplementary Fig. 5E), were significantly lower in the ischemic hindlimb of KO mice compared to that of WT mice. However, the anti-angiogenic miRNAs -92a and -206 (Supplementary Fig. 5C-D), and the pro-survival proteins BCL-2 and pAKT (Supplementary Fig. 5F, G), were not different between KO and WT mice.

Role of exogenous ghrelin for improving the revascularization response to HLI in diabetes 
Having confirmed the significant role of endogenous ghrelin for driving the revascularization response following $\mathrm{HLI}$, and considering that circulating levels of ghrelin are adversely reduced in diabetic mice, we next tested the efficacy of exogenous ghrelin (both AG and DAG) to promote revascularization and angiogenesis in diabetic $\mathrm{db} / \mathrm{db}$ mice with $\mathrm{HLI}$.

\section{Recovery of hind limb blood flow following HLI in diabetes - ghrelin therapy}

The daily administration of AG to both DM and ND mice significantly improved perfusion recovery over the 14 days post-HLI, such that laser Doppler imaging showed that limb perfusion of treated DM mice was similar to ND mice (Fig. 4A-B). However, DAG did not significantly improve blood flow recovery at day 14 in DM mice, although it did improve recovery in ND mice.

AG treatment to both ND and DM mice significantly augmented the increase in vascular volume associated with $\mathrm{HLI}$, compared to vehicle alone (micro-CT; Fig. 4C-D), as well as augmenting the increase in capillary and arteriole density (a-smooth muscle actin staining; Fig. 4E-G). Although DAG increased vascular volume (Fig. 4C-D) and arteriole density above vehicle alone (Fig. 4G), it did not enhance capillary density in ND and DM mice (Fig. 4F).

Collectively, the actions of AG in diabetes to improve blood perfusion, vascular volume, and capillary/arteriole formation in the ischemic hindlimb, likely underpins the improved functional capacity compared to untreated DM mice (Supplementary Fig. 6).

\section{Ghrelin therapy improves vascular function during diabetic HLI}

Diabetes was associated with impaired vascular function within the ischemic limb following HLI (Supplementary Fig. 7). We next assessed the ability of ghrelin (AG and DAG) to preserve vascular function in diabetic $\mathrm{db} / \mathrm{db}$ mice following HLI using micoangiography. The vasodilatory response of the 50-150 $\mu \mathrm{m}$ vessels to ACh was impaired by HLI, diabetes or the combination of both (Supplementary Fig. 7B). Remarkably, AG treatment in DM+HLI mice improved the vascular response to ACh so that it was essentially indistinguishable from control mice (i.e. ND+Sham; 40\% increase in ID of the 50-100 $\mu \mathrm{m}$ vessels; Supplementary Fig. 7B). In comparison, DAG did not improve the blunted responses to ACh, greater than that of vehicle alone, in either the DM or ND mice. The vasodilatory response to SNP did not appear to be compromised by either HLI or diabetes, such that the magnitude of vasodilation was comparable between all ND and DM groups (Supplementary Fig. 7C).

The vasodilatory responses to Rho-kinase inhibition (Fasudil), particularly of the 50-150 $\mu \mathrm{m}$ sized vessels, appeared to be exacerbated only in diabetic mice with or without accompanying $\mathrm{HLI}$ ( $40 \pm 7 \%$ increase in ID for the 50-100 $\mu \mathrm{m}$ vessels; Supplementary Fig. 7D). Importantly, AG administration to diabetic mice normalized the vasodilatory response to Fasudil, similar to ND+Sham mice (Supplementary Fig. 7D).

\section{Ghrelin therapy enhances cell survival and decreases fibrosis}


Diabetes was associated with a significant increase in both total $\mathrm{TUNEL}^{+}$apoptotic cells (Fig. 5A-B) and TUNEL ${ }^{+}$endothelial cells (TUNEL and isolectin-B4 positive; Fig. 5C-D), which was further exacerbated following 14 days of HLI. AG therapy was able to significantly reduce the adverse increase in total TUNEL $^{+}$apoptotic and TUNEL ${ }^{+}$endothelial cells, particularly in DM mice (Fig. 5B-D). In comparison, DAG did not attenuate the increase in total TUNEL ${ }^{+}$apoptotic cells (Fig. 5B), but it did attenuate the increase in TUNEL $^{+}$endothelial cells (Fig. 5D).

HLI-induced injury was also associated with muscle collagen deposition, i.e. fibrosis (Fig. 5E), which was significantly worse in DM mice compared to ND mice (Fig. 5F). Treatment with AG and DAG was effective in reducing the severity of $\mathrm{HLI}$-induced fibrosis in DM and ND mice, although the anti-fibrotic effects were more pronounced with AG compared to that of DAG (Fig. 5F).

\section{Ghrelin treatment activates pro-angiogenic miRNAs}

In ND mice, both AG and DAG treatment following HLI significantly increased the expression of proangiogenic miRNAs -126 and -132 , compared to vehicle treatment, whilst also suppressing the HLIinduced overexpression of anti-angiogenic miRNAs -206 and -92a (Fig. 6A-D). Importantly, the effect of $A G$ and DAG on miRNA expression was associated with the observed increase in the expression of VEGF, pAKT and Bcl-2 proteins (Fig. 6E-G).

In DM mice, the small increase in miRNA-126 and -132 expression following AG treatment was not significant, although AG did suppress over-expression of miRNA-206 (Fig. 6D) and, importantly, increased VEGF, pAKT and Bcl-2 protein expression (Fig. 6E-G). In contrast, DAG was ineffective in modulating any of the HLI-induced changes in miRNAs or the associated target proteins (Fig. 6A-G).

\section{Discussion}

Diabetes remains one of the greatest risk factors for the onset of PAD, which is associated with an unacceptably high premature mortality rate ${ }^{28}$. Restoring or increasing perfusion to alleviate ischemia has been the goal of clinical research for several decades. However, potentially promising experimental strategies for promoting functional angiogenesis have often failed to reach routine clinical application. Here, we provide novel data identifying the important role of endogenous ghrelin, which is dysregulated in T2DM, for driving the angiogenic response following ischemia to improve limb perfusion. Moreover, exogenous ghrelin treatment appears to improve revascularization, at least in the diabetic $\mathrm{db} / \mathrm{db}$ mouse model, that may be mediated via the modulation of key vascular-specific miRNAs $-126,-132,-92 \mathrm{a}$, and $-206$.

Ghrelin is derived from preproghrelin, a 117 amino-acid precursor that is predominantly produced by $\mathrm{P} / \mathrm{D} 1$-like cells in the oxyntic glands of the gastric mucosa ${ }^{29}$. Acylated ghrelin and its receptor (GHS-R) are ubiquitous throughout the cardiovascular system ${ }^{29,30}$, suggesting ghrelin has a prominent role in 
cardiovascular homeostasis ${ }^{31}$. To date, a growing body of literature advocates the beneficial effects of $A G$ in maintaining cardiovascular health ${ }^{6,32,33}$.

Clinically, endogenous levels of ghrelin appear to be downregulated in diabetic patients ${ }^{15}$, an observation confirmed in this study. Herein, we further show that both diabetic and non-diabetic patients with PAD had low circulating levels of acylated ghrelin (below a critical level of $<67 \mathrm{pg} / \mathrm{mL}$ ). These results may suggest that dysregulation of endogenous ghrelin, rather than diabetes per se, may be the crucial risk factor for developing PAD. Hence, low plasma levels of endogenous AG in the clinic may serve at the very least as a prognostic marker for PAD progression.

Experimental data in this study showed that the magnitude of damage to the hindlimb microvasculature following prolonged ischemia was essentially the same for diabetic $\mathrm{db} / \mathrm{db}$ mice, which had only $\sim 7 \%$ on the circulating ghrelin levels compared to non-diabetic mice, and ghrelin knock out mice, which lacked diabetes. These observations once again strengthen the idea that dysregulation of endogenous ghrelin, which is a consequence of diabetes, underpins the development of PAD.

Role of miRNAs for mediating angiogenesis following $\mathrm{HLI}$

The mechanism(s) by which ghrelin dysregulation underpins impaired vascular reparation and angiogenesis remains unclear. We have previously reported that the expression of pro-angiogenic miRNAs -126 and -132 , which were correlated to circulating ghrelin levels, appeared to determine the severity of hindlimb vascular damage following $\mathrm{HLI}$, at least in a young, healthy mouse model ${ }^{11}$. Togliatto et al. also reported that increasing circulating levels of des-acylated ghrelin promoted skeletal muscle regeneration via the modulation of miRNAs $-221 \&-222^{34}$, and protected the hindlimb vasculature against reactive oxygen species-mediated damage, through the modulation of miRNA-126 35 .

In this study, we aimed to more closely emulate the clinical setting and, thus, report the efficacy of acylated ghrelin treatment in an aged, diabetic murine model of PAD. We report that the downregulation of miRNA-126 and -132, in both the diabetic and ghrelin KO mouse model mirrored the level of endogenous ghrelin and importantly, was closely associated with the impaired vascular reparative ability following HLI. The protein targets for miRNA-126, SPRED1 and PIK3R2, are both negative regulators of VEGFA ${ }^{36}$. Moreover, miRNA-132 targets p120RasGap and Ras-activation, which are known inhibitors of angiogenesis ${ }^{37}$. Hence, it is mechanistically plausible that the modulation of select miRNAs may underpin the pathway by which ghrelin facilitates its vascular-protective effects, independent of diabetes, although further mechanistic studies are essential to confirm this hypothesis.

\section{Therapeutic potential of exogenous ghrelin for HLI}

In this study, we observed that daily AG administration following $\mathrm{HLI}$ in diabetic $\mathrm{db} / \mathrm{db}$ mice dramatically improved hindlimb blood flow perfusion recovery, through improved vascular function, increased cell survival, angiogenesis/arteriogenesis, and skeletal tissue architecture recovery. Interestingly, DAG also exhibited some beneficial effects but consistently to a lesser extent than AG treatment. We also observed 
that these beneficial vascular effects driven by AG were associated with modulation of specific miRNAs. These results once again suggest that ghrelin may elicit its vascular effects, at least in part, through the modulation of miRNAs.

Arpino et al. ${ }^{38}$ demonstrated that vascular regeneration following HLI constitutes a highly flawed vascular network, with an impaired vasomotor control system. In agreement, we were able to show, with the use of high-resolution microangiography, that the impaired vascular regeneration response to HLI observed in ghrelin $\mathrm{KO}$ mice and diabetic $\mathrm{db} / \mathrm{db}$ mice was associated with vascular endothelial dysfunction, based on the blunted vasodilatory responses to ACh. Remarkably, however, ghrelin therapy to diabetic mice not only amplified the angiogenic response to $\mathrm{HLI}$, but endothelial function of these newly formed vessels was functionally normal (compared to ND sham animals).

The hindlimb vasculature of diabetic sham mice had a blunted vasodilatory response to ACh, which we have also recently demonstrated in the coronary vasculature of diabetic $\mathrm{db} / \mathrm{db}$ mice ${ }^{39}$. Importantly, considering that ghrelin treatment to diabetic sham animals restored vascular function to a level that was indistinguishable from their ND counterparts, it is likely that dysregulation of ghrelin in diabetes underpins the observed endothelial dysfunction in diabetes.

\section{Conclusion}

In summary, endogenous ghrelin plays an important role in driving the crucial revascularization response following ischemia. Clinically, human patients with diabetes with or without PAD appear to have reduced levels of AG. We also demonstrate that exogenous AG treatment in a diabetic murine model of PAD promotes functional vascularization, likely through the modulation of specific angiogenic miRNAs. Ultimately, this study highlights the future potential of exogenous AG as a novel therapeutic intervention for the adjunct treatment of PAD in both diabetic and non-diabetic patients.

\section{Abbreviations}

$A B I-$ Ankle-brachial index

ABP - Arterial blood pressure

ACh - Acetylcholine

AG - Acylated ghrelin

DAG - Des-acylated ghrelin

DM Veh - Diabetes mellitus with vehicle treatment

GHSR1a - Growth hormone secretagogue receptor 1a 
$\mathrm{HLI}$ - Hind limb ischemia

HR - Heart rate

KO - Knock out

MABP - Mean arterial blood pressure

miRNAs - microRNAs

ND - Non-diabetic

PAD - Peripheral arterial disease

T2DM - Type 2 diabetes mellitus

TUNEL - Terminal deoxynucleotidyl transferase mediated dUTP nick-end labelling

VEGFA - Vascular endothelial growth factor A

\section{Declarations}

\section{Ethics approval and consent to participate}

Human Ethical approval was obtained from the University of Otago Human Ethics Committee (Health) (H17/012), and the study conformed to the standards set by the Declaration of Helsinki.

All animal experimentation was approved by the Animal Ethics Committee of the University of Otago, New Zealand, and the National Cerebral and Cardiovascular Centre, Japan. All procedures are reported in accordance with the ARRIVE guidelines.

\section{Consent for publication}

Not applicable.

\section{Availability of data and materials}

The datasets used and/or analysed during the current study are available from the corresponding author on reasonable request..

\section{Competing interests}

The authors declare that they have no competing interests.

\section{Funding}


This work was funded by a Royal Society of New Zealand Catalyst Seeding grant (CSG-U001603), the School of Biomedical Sciences Deans Bequest, Otago University, New Zealand, a New Zealand Lottery Health Research grant (R-LHR-2016-27043) and a Grant-in-Aid for Scientific Research (KAKEN 19H03405) and an intramural grant of the NCVC 25-3-1.

\section{Authors' contributions}

All authors contributed to the interpretation of data and reviewing of the manuscript. JPHN performed the majority of animal experimental work, data analysis, and drafted the manuscript; JTP and HT lead and contributed to the angiography experiments provided guidance; JTP further contributed to the editing of the manuscript drafts; $\mathrm{HH}, \mathrm{MK}$ and TS provided input and direction with the use of ghrelin and $\mathrm{KO}$ mice for HLI; KT, GJ and AVR lead the clinical component of the study; PL contributed to the laser Doppler experiments. $D C$ contributed to the molecular analysis, $A D$ and $L J D$ performed the functional assessments. DOS and RK conceived, designed and supervised the study, and wrote the final version of the manuscript.

\section{Acknowledgements}

The authors are grateful to Andrew McNaughton for his work in supporting the micro-CT studies. We would like to thank Dr Ash Gillon for this help in microscopy and data analysis.

\section{References}

1. Ouriel, K. Peripheral arterial disease. Lancet 358, 1257-1264 (2001).

2. Norgren, L. et al. Inter-Society Consensus for the management of peripheral arterial disease (TASC II). Int. Angiol. 26, 82-157 (2007).

3. Song, P. et al. Articles Global , regional , and national prevalence and risk factors for peripheral artery disease in 2015: an updated systematic review and analysis. Lancet Glob. Heal. 7, e1020-e1030 (2019).

4. Shah, A. D. et al. Type 2 diabetes and incidence of cardiovascular diseases: A cohort study in $1 \cdot 9$ million people. Lancet Diabetes Endocrinol. 3, 105-114 (2015).

5. Reinecke, H. et al. Peripheral arterial disease and critical limb ischaemia: Still poor outcomes and lack of guideline adherence. Eur. Heart J. 36, 932-938 (2015).

6. Neale, J. P. H., Pearson, J. T., Katare, R. \& Schwenke, D. O. Ghrelin, MicroRNAs, and Critical Limb Ischemia: Hungering for a Novel Treatment Option. Front. Endocrinol. (Lausanne). 8, 350 (2017).

7. Kojima, M. et al. Ghrelin is a growth-hormone-releasing acylated peptide from stomach. Nature $\mathbf{4 0 2}$, 656-660 (1999).

8. Li, A., Cheng, G., Zhu, G. hui \& Tarnawski, A. S. Ghrelin stimulates angiogenesis in human microvascular endothelial cells: Implications beyond GH release. Biochem. Biophys. Res. Commun. 353, 238-243 (2007). 
9. Ahluwalia, A., Li, A., Cheng, G., Deng, X. \& Tarnawski, A. S. Reduced ghrelin in endothelial cells plays important mechanistic role in aging-related impairment of angiogenesis. J. Physiol. Pharmacol. 60, 29-34 (2009).

10. Yuan, M. J. et al. Myocardial angiogenesis after chronic ghrelin treatment in a rat myocardial infarction model. Regul. Pept. 179, 39-42 (2012).

11. Katare, R. et al. Ghrelin Promotes Functional Angiogenesis in a Mouse Model of Critical Limb Ischemia Through Activation of Proangiogenic MicroRNAs. Endocrinology 157, 432-445 (2015).

12. Wang, L., Chen, Q., Li, G. \& Ke, D. Ghrelin ameliorates impaired angiogenesis of ischemic myocardium through GHSR1a-mediated AMPK/eNOS signal pathway in diabetic rats. Peptides 73, 77-87 (2015).

13. Rajan, D. et al. Human Ghrelin Protects Animals from Renal Ischemia- Reperfusion Injury through the Vagus Nerve. Surgery 151, 37-47 (2013).

14. Prodam, F. \& Filigheddu, N. Ghrelin Gene Products in Acute and Chronic Inflammation. Arch. Immunol. Ther. Exp. (Warsz). 62, 369-384 (2014).

15. Churm, R., Davies, J. S., Stephens, J. W. \& Prior, S. L. Ghrelin function in human obesity and type 2 diabetes: a concise review. Obes. Rev. (2016). doi:10.1111/obr.12474

16. Bartel, D. P. MicroRNAs: Genomics, Biogenesis, Mechanism, and Function. Cell 116, 281-297 (2004).

17. Delhanty, P. J. D. et al. The acylated (AG) to unacylated (UAG) ghrelin ratio in esterase inhibitortreated blood is higher than previously described. Clin. Endocrinol. (Oxf). 82, 142-146 (2015).

18. Lotfi, S. et al. Towards a more relevant hind limb model of muscle ischaemia. Atherosclerosis 227, 1-8 (2013).

19. Jackson, S. J. et al. Does age matter? The impact of rodent age on study outcomes. Lab. Anim. 51, 160-169 (2017).

20. Caporali, A. et al. p75NTR-dependent activation of NF-KB regulates microRNA-503 transcription and pericyte-endothelial crosstalk in diabetes after limb ischaemia. Nat. Commun. 6, 8024 (2015).

21. Lu, Q. et al. Angiogenic Factor AGGF1 Promotes Therapeutic Angiogenesis in a Mouse Limb Ischemia Model. PLoS One 7, e46998 (2012).

22. Sonobe, T., Tsuchimochi, H., Schwenke, D. O., Pearson, J. T. \& Shirai, M. Treadmill running improves hindlimb arteriolar endothelial function in type 1 diabetic mice as visualized by X-ray microangiography. Cardiovasc. Diabetol. 14, 51 (2015).

23. Spinetti, G. et al. MicroRNA-15a and MicroRNA-16 impair human circulating proangiogenic cell functions and are increased in the proangiogenic cells and serum of patients with critical limb ischemia. Circ. Res. 112, 335-346 (2013).

24. Katare, R., Caporali, A., Emanueli, C. \& Madeddu, P. Benfotiamine improves functional recovery of the infarcted heart via activation of pro-survival G6PD/Akt signaling pathway and modulation of neurohormonal response. J. Mol. Cell. Cardiol. 49, 625-638 (2010).

25. Hosoda, H. et al. Optimum Collection and Storage Conditions for Ghre- lin Measurements: Octanoyl Modification of Ghrelin Is Rapidly Hydrolyzed to Desacyl Ghrelin in Blood Samples. Clin. Chem. 50, 
1077-1080 (2004).

26. He, Y. et al. Critical function of Bmx / Etk in ischemia- mediated arteriogenesis and angiogenesis. J. Clin. Invest. 116, 2344-2355 (2006).

27. Nass, R. et al. Age-dependent decline in acyl-ghrelin concentrations and reduced association of acylghrelin and growth hormone in healthy older adults. J. Clin. Endocrinol. Metab. 99, 602-608 (2014).

28. Zheng, Y., Ley, S. H. \& Hu, F. B. Global aetiology and epidemiology of type 2 diabetes mellitus and its complications. Nat. Rev. Endocrinol. 14, 88-98 (2018).

29. Gnanapavan, S. et al. The Tissue Distribution of the mRNA of Ghrelin and Subtypes of Its Receptor, GHS-R, in Humans. J. Clin. Endocrinol. Metab. 87, 2988-2991 (2002).

30. Kleinz, M. J., Maguire, J. J., Skepper, J. N. \& Davenport, A. P. Functional and immunocytochemical evidence for a role of ghrelin and des-octanoyl ghrelin in the regulation of vascular tone in man. Cardiovasc. Res. 69, 227-235 (2006).

31. Nagaya, N. et al. Hemodynamic and hormonal effects of human ghrelin in healthy volunteers. Am J Physiol Regul Integr Comp Physiol 280, R1483-7 (2001).

32. Lilleness, B. M. \& Frishman, W. H. Ghrelin and the Cardiovascular System. Cardiol. Rev. 24, 288-297 (2016).

33. Tokudome, T., Otani, K., Miyazato, M. \& Kangawa, K. Ghrelin and the Heart. Peptides 111, 42-46 (2019).

34. Togliatto, G. et al. Unacylated ghrelin promotes skeletal muscle regeneration following hindlimb ischemia via SOD-2-mediated miR-221/222 expression. J Am Hear. Assoc 2, e000376 (2013).

35. Togliatto, G. et al. Unacylated ghrelin induces oxidative stress resistance in a glucose intolerance and peripheral artery disease mouse model by restoring endothelial cell miR-126 expression. Diabetes 64, 1370-1382 (2015).

36. Fish, J. E. et al. miR-126 Regulates Angiogenic Signaling and Vascular Integrity. Dev. Cell 15, 272284 (2008).

37. Anand, S. et al. MicroRNA-132-mediated loss of p120RasGAP activates the endothelium to facilitate pathological angiogenesis. Nat. Med. 16, 909-914 (2010).

38. Arpino, J. M. et al. Four-Dimensional Microvascular Analysis Reveals That Regenerative Angiogenesis in Ischemic Muscle Produces a Flawed Microcirculation John-Michael. Circ. Res. 120, 1453-1465 (2017).

39. Katare, R. et al. Progressive Decrease in Coronary Vascular Function Associated With Type 2 Diabetic Heart Disease. Frontiers in Physiology 9, 696 (2018).

\section{Tables}

Table 1 | Participant demographics 


\begin{tabular}{|c|c|c|c|c|}
\hline - & $\begin{array}{l}\text { Controls } \\
(\underline{n=30}) .\end{array}$ & $\begin{array}{l}\underline{\text { T2DM }} \\
(\underline{n=25})\end{array}$ & $\begin{array}{l}\underline{P A D} \\
(n=41) .\end{array}$ & $\begin{array}{l}\frac{P A D+T 2 D}{(n=26)} . \\
(\underline{n}=10\end{array}$ \\
\hline Males (\%) & $17(57)$ & $12(48)$ & $30(73)$ & $18(69)$ \\
\hline Age (y) & $61.1 \pm 6.7$ & $63.6 \pm 8.2$ & $71.9 \pm 10.7 *^{\dagger}$ & $72.8 \pm 9.0 *^{\dagger}$ \\
\hline $\mathrm{BMI}\left(\mathrm{kg} / \mathrm{m}^{2}\right)$ & $28.3 \pm 5.1$ & $32.3 \pm 7.2$ & $25.9 \pm 5.1^{\dagger}$ & $29.3 \pm 5.9$ \\
\hline $\mathrm{HbA} 1 \mathrm{c}(\mathrm{mmol} / \mathrm{mol})$ & $\begin{array}{l}35(33- \\
37)\end{array}$ & $64(51-77)$ & $\underbrace{38}_{\star \neq}(35-41)$ & $\underset{\star}{57}(45-63)$ \\
\hline Resting $A B I$ in lower limb & $1.1 \pm 0.1$ & $1.1 \pm 0.1$ & $0.6 \pm 0.2 \star^{\dagger}$ & $0.6 \pm 0.3 *^{\dagger}$ \\
\hline $\begin{array}{l}\text { Smoking status: } \mathrm{n}=\text { current } / \mathrm{ex} / \\
\text { never }\end{array}$ & $1 / 8 / 21$ & $0 / 10 / 15$ & $17 / 17 / 7$ & $3 / 19 / 4$ \\
\hline $\begin{array}{l}\text { T2DM, Type } 2 \text { Diabetes Mellitus; } P A L \\
\text { glycosylated haemoglobin, } A B I \text {, ankI } \\
\text { is shown as median (IQR); The catec } \\
\text { control group; } †<<0.05 \text { versus } T 2 D\end{array}$ & $\begin{array}{l}\text { oheral Arte } \\
\text { hial index. } \\
\text { data is pre } \\
\text { up; } ¥ \mathrm{P}<0\end{array}$ & $\begin{array}{l}\text { Disease; } B M \\
\text { e, BMI and Al } \\
\text { nted as numb } \\
\text { versus PAD }\end{array}$ & $\begin{array}{l}\text { dy mass inde } \\
\text { e shown as } \mathrm{m} \\
\text { eercentage). }\end{array}$ & $\begin{array}{l}\text { bA1c, } \\
\pm \text { SD; HbA1C, } \\
0.05 \text { versus }\end{array}$ \\
\hline
\end{tabular}

Table 2 | Ghrelin parameters

\begin{tabular}{lllll} 
& Controls $(\mathrm{n}=30)$ & T2DM $(\mathrm{n}=25)$ & $\mathrm{PAD}(\mathrm{n}=41)$ & $\mathrm{PAD}+\mathrm{T} 2 \mathrm{D}(\mathrm{n}=26)$ \\
\hline $\mathrm{AG}(\mathrm{pg} / \mathrm{mL}) *$ & $87(70-136)$ & $46(36-96)$ & $70(28-114)$ & $46(25-116)$ \\
\hline $\mathrm{DAG}(\mathrm{pg} / \mathrm{mL})$ & $116(71-129)$ & $67(46-103)$ & $92(47-155)$ & $96(29-176)$ \\
\hline AG:DAG ratio & $0.90(0.74-1.11)$ & $0.84(0.52-1.16)$ & $0.74(0.36-1.43)$ & $0.64(0.37-1.20)$ \\
\hline $\begin{array}{l}\text { Total G } \\
(\mathrm{pg} / \mathrm{mL})\end{array}$ & $209(145-695)$ & $126(87-202)$ & $175(85-251)$ & $147(59-334)$ \\
\hline
\end{tabular}

T2DM, Type 2 Diabetes Mellitus; PAD, Peripheral Arterial Disease; AG, acylated ghrelin; DAG, deacylated ghrelin; Total G, total ghrelin. AG, DAG, AG:DAG ratio and Total $G$ are shown as median (IQR). * $P=0.049$ for a difference between groups.

Table 3 | Cut off values calculated using the Youden index from the receiver operating characteristic (ROC) curve 


\begin{tabular}{llllll} 
Group & Cut-off value $(\mathrm{pg} / \mathrm{mL})$ & Sensitivity $(\%)$ & Specificity $(\%)$ & AUROC & AUROC $95 \% \mathrm{Cl}$ \\
\hline T2D & 75 & 72 & 73 & 0.71 & $0.57-0.82$ \\
\hline PAD & 67 & 49 & 80 & 0.63 & $0.51-0.75$ \\
\hline PAD+T2DM & 76 & 69 & 73 & 0.68 & $0.54-0.80$
\end{tabular}

T2DM, Type 2 Diabetes Mellitus; PAD, Peripheral Arterial Disease; AUROC, area under the ROC curve.

Table 4 | Logistic regression analysis to calculate the odds ratios for having acylated ghrelin (AG) below the threshold $67 \mathrm{pg} / \mathrm{mL}$

\begin{tabular}{lllllll} 
Group & \multicolumn{2}{l}{ Unadjusted odds ratio } & \multicolumn{4}{l}{ Adjusted for age } \\
& Odds ratio & $95 \% \mathrm{Cl}$ & p-value & Odds ratio & $95 \% \mathrm{Cl}$ & p-value \\
\hline T2DM: & 6.00 & $1.81-19.93$ & 0.003 & 5.82 & $1.74-19.42$ & 0.004 \\
$\mathrm{AG}<67 \mathrm{pg} / \mathrm{mL}$ & & & & & & \\
\hline $\mathrm{PAD}:$ & 3.46 & $1.17-10.22$ & 0.025 & 2.92 & $0.90-9.48$ & 0.075 \\
$\mathrm{AG}<67 \mathrm{pg} / \mathrm{mL}$ & & & & & & \\
\hline $\mathrm{PAD}+\mathrm{T} 2 \mathrm{DM}:$ & 6.40 & $1.94-21.11$ & 0.002 & 5.32 & $11.47-19.12$ & 0.011 \\
$\mathrm{AG}<67 \mathrm{pg} / \mathrm{mL}$ & & & & & &
\end{tabular}

The reference population was the control group. The adjusted model includes age. Adjusting for sex had no effect (data not shown). T2DM, Type 2 Diabetes Mellitus; PAD, Peripheral Arterial Disease.

\section{Figures}



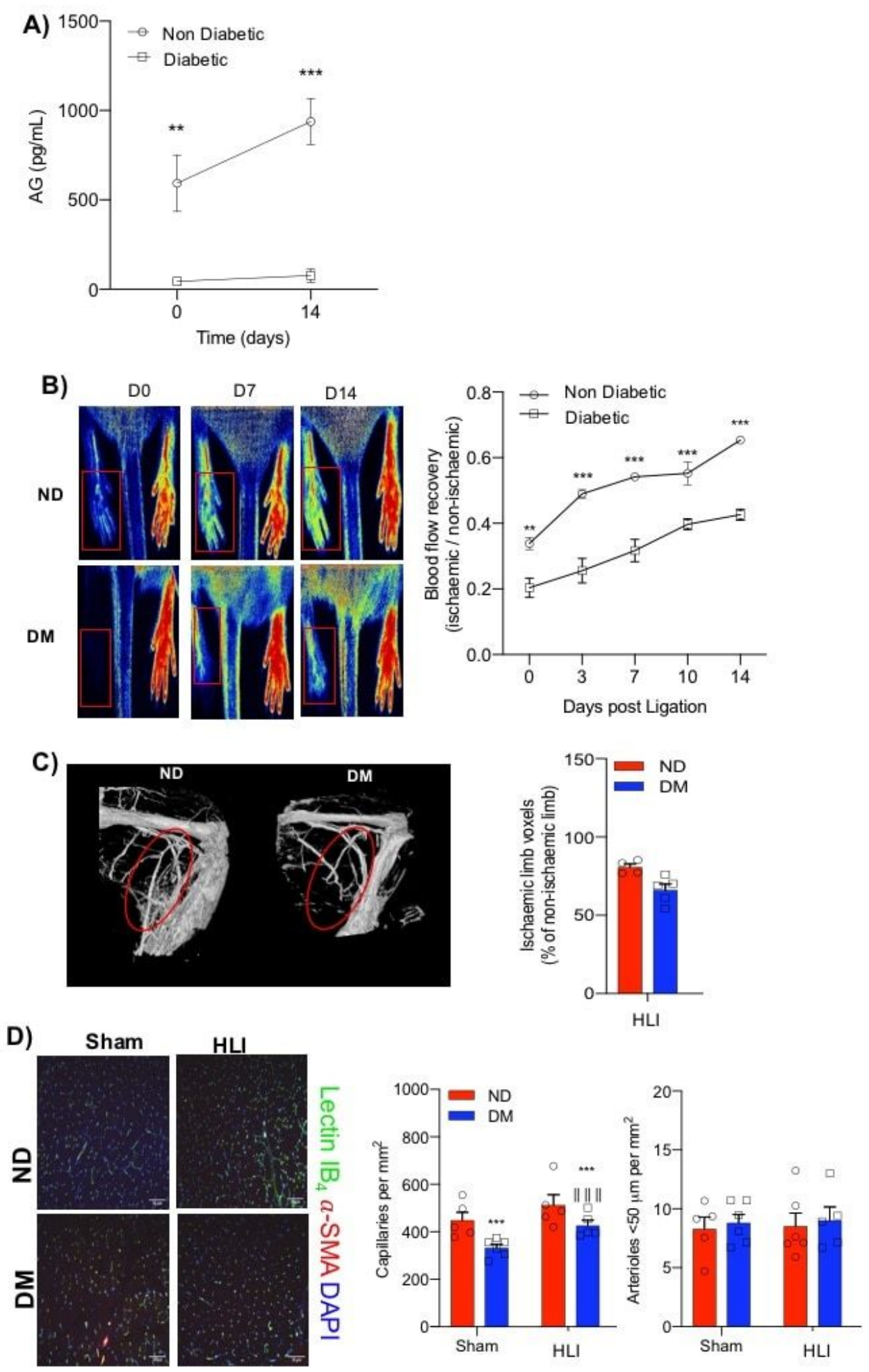

\section{Figure 1}

Circulating acylated ghrelin (AG) is reduced in diabetic $\mathrm{db} / \mathrm{db}$ mice. A) A comparison of plasma AG between non-diabetic (ND) and diabetic (DM) mice before and after 14 days of hindlimb ischemia (HLI; $\mathrm{n}=6$ ). B) Representative laser Doppler images of ND and DM mice 1 hour after HLI (Day 0), and then 7 and 14 days after HLI. Red squares delimit the area measured in the ischemic foot. Data are quantified in the adjoining line graph showing the time course of blood flow recovery (calculated as the ratio of blood 
flow in ischemic to contralateral foot; $n=5-6)$. C) Representative micro-CT images showing the hindlimb vasculature in ND and DM mice after 14 days of HLI. The red oval indicates the region of interest for quantifying vascular volume, as presented in the adjoining bar graph ( $n=5-6$ for all study groups). D) Representative ischemic gastrocnemius sections stained with the endothelial marker isolectin B4 (green fluorescent) and a-smooth muscle actin (red fluorescent) in the arterial wall (scale bar, $50 \mu \mathrm{m}$ ) of ND and DM mice 14 days after HLI. Both capillary and arteriole density (diameter $<50 \mu \mathrm{m}$ ) are quantified in the adjoining bar graphs $(n=5-6)$. Data are presented as mean \pm S.E.M. Statistical comparisons were made using two-way ANOVA, followed by Sidak multiple comparisons post hoc tests, or unpaired t-tests. * Significant difference between ND and DM (** $P<0.01$, $* \star * P<0.001)$; $\|$ Significant difference between Sham and HLI (II II || P < 0.001). 


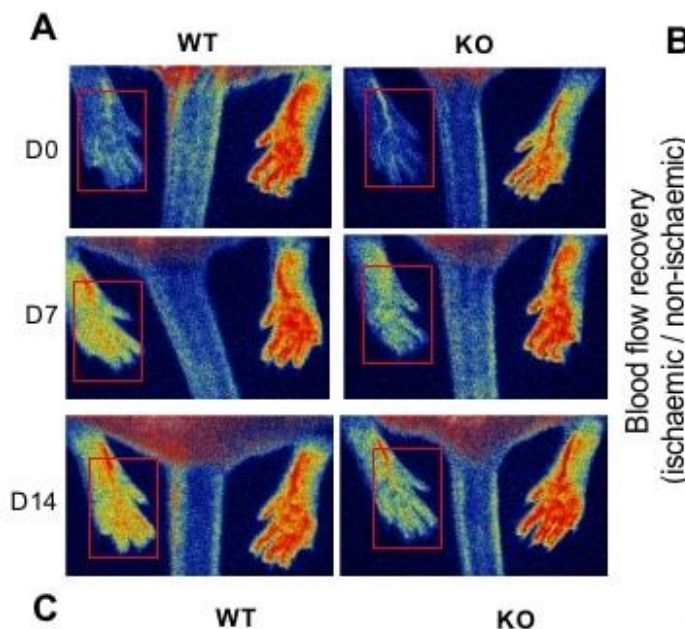

B
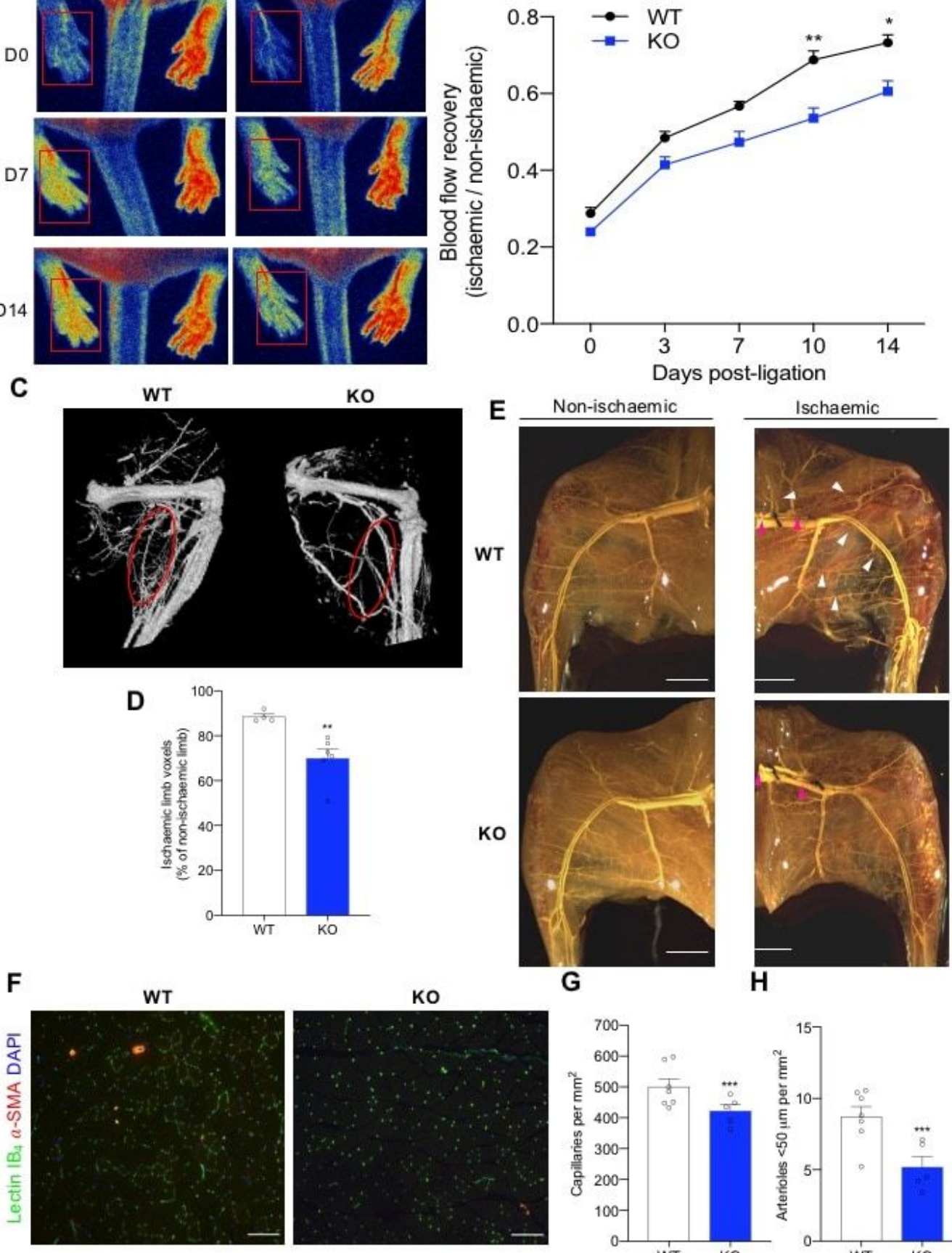

ко
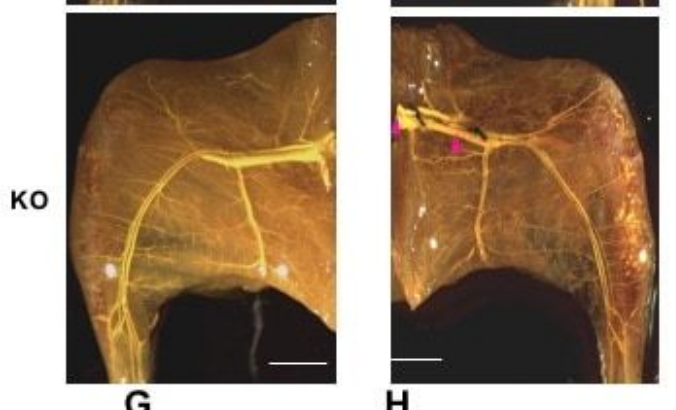

G

$\mathbf{H}$

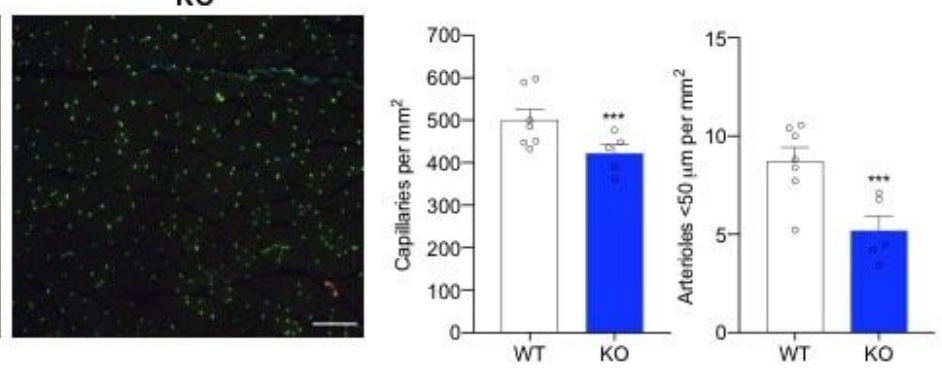

\section{Figure 3}

Restoration of blood flow after hindlimb ischemia is impaired in ghrelin knockout mice A) Representative laser Doppler images at baseline, day 7, and day 14 following HLI in KO and WT mice. Red squares delimit the area measured in the ischemic foot. B) Time course of blood flow recovery calculated as the ratio of blood flow in ischemic to contralateral foot $(n=7-10)$. C, Representative micro-CT images are showing the hindlimb vasculature in KO and WT mice undergoing HLI for 14 days. D, Quantification of 
vascular volume by micro-CT analysis $(n=4-6)$. E, Representative whole-mount images of Microfil vascular cast limbs. White arrow heads indicate enlarged arteries, pink arrow heads indicate ligations. $\mathrm{F}$, Representative ischemic gastrocnemius sections stained with the endothelial marker, isolectin-B4 (green

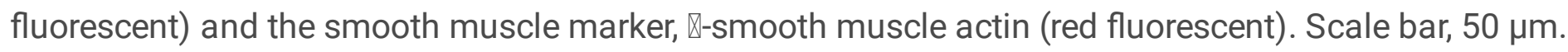
Quantification of $(\mathrm{G})$ capillary and $(\mathrm{H})$ arteriole density (diameter $<50 \mu \mathrm{m}$ ) density at day 14 post HLI $(n=5-7)$. Data are presented as mean \pm S.E.M. Statistical comparisons were made by using a RM twoway ANOVA, followed by Sidak multiple comparisons test, $(B)$ two-tailed unpaired Student t test $\left(E_{,}, G_{1}\right)$, or two-tailed unpaired Mann-Whitney $U$ test $(H)$. * Significantly different from WT counterpart $(* P<0.05$, ** $P<0.01, \star \star \star P<0.001)$. 


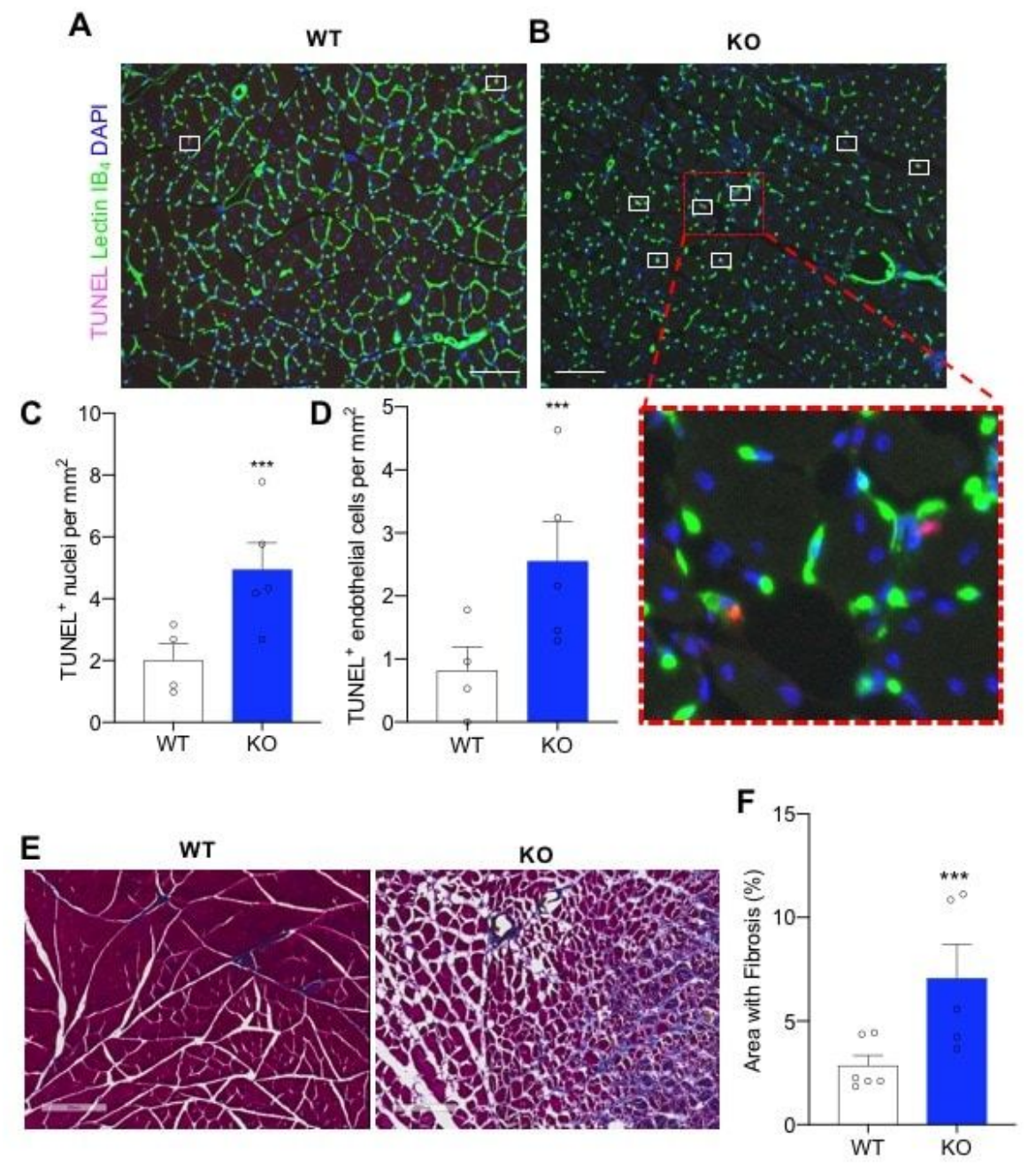

\section{Figure 5}

Deficiency of endogenous ghrelin promotes apoptosis and fibrosis after hind limb ischemia A) Representative images of TUNEL-positive myocytes and (B) endothelial cells in the ischemic gastrocnemius muscle, 14 days post-HLI C) Quantification of TUNEL-positive myocytes and (D) endothelial cells represented per $\mathrm{mm} 2(\mathrm{n}=4-5)$. Scale bars, $50 \mu \mathrm{m}$. E) Representative histological images of Masson's trichrome staining in the ischemic gastrocnemius muscle of WT and KO mice 14 days post- 
HLI. F) Quantification of gastrocnemius muscle fibrosis ( $n=5-6)$. Scale bars, $200 \mu \mathrm{m}$. Data are presented as mean \pm S.E.M. Statistical comparisons were made by using a two-tailed unpaired Mann-Whitney $U$ test $(C, D, F) .{ }^{*}$ Significantly different from WT counterpart $(* \star * P<0.001)$.
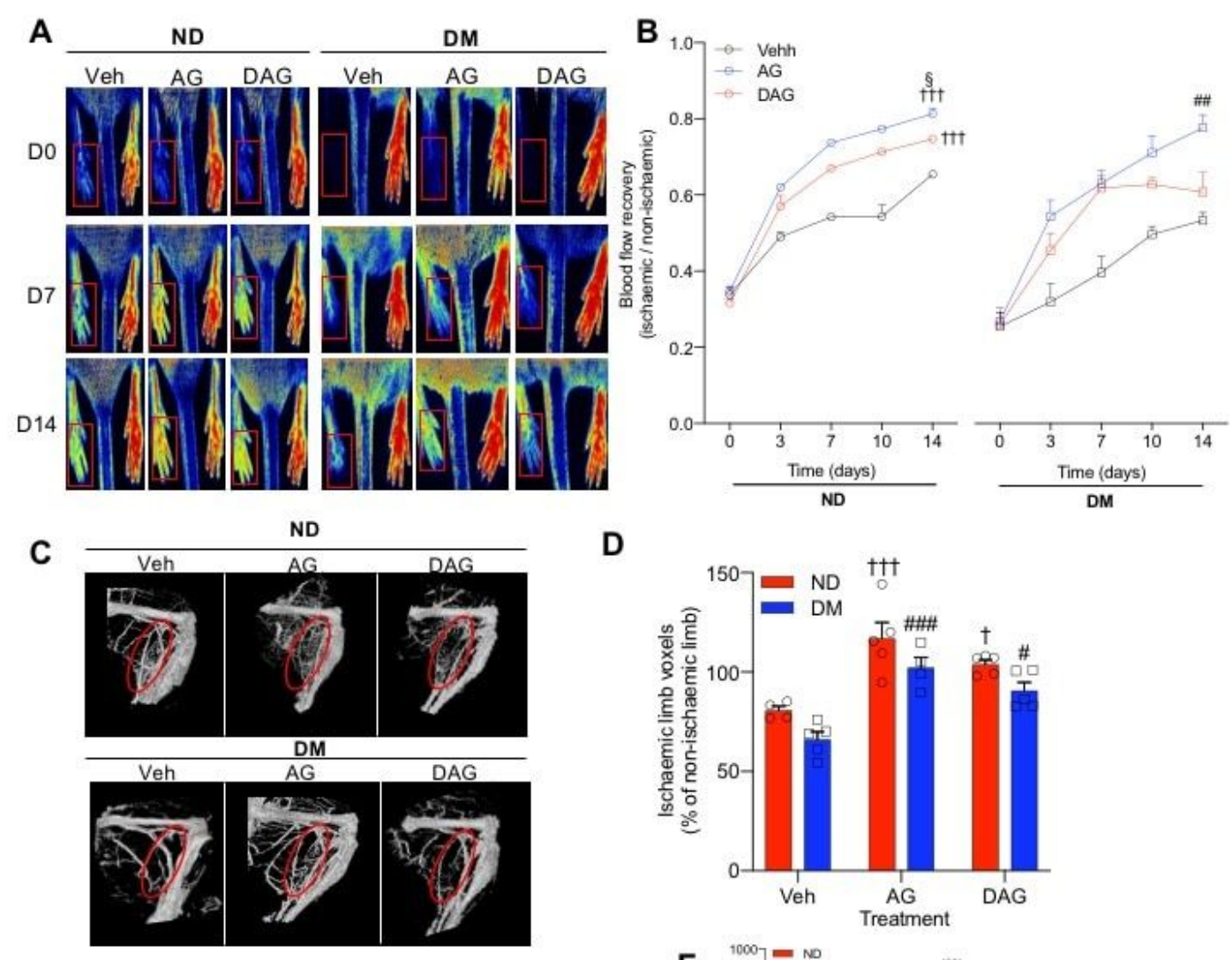

D
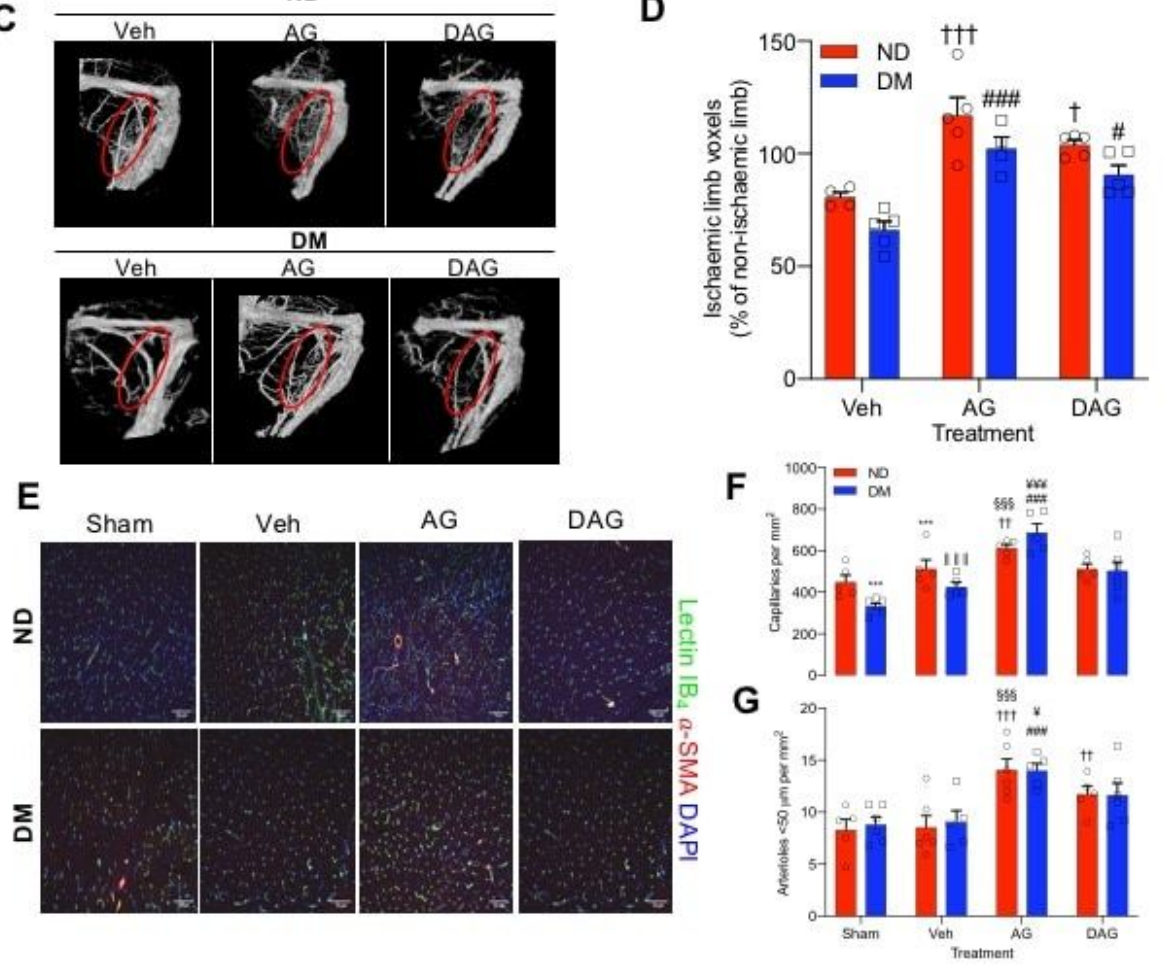

\section{Figure 8}

Exogenous ghrelin enhances tissue perfusion after hind limb ischemia in diabetic $\mathrm{db} / \mathrm{db}$ mice A) Representative laser Doppler images of ND and DM mice undergoing HLI and treated with vehicle, AG, or 
DAG for 14 days. Red squares delimit the area measured in the ischemic foot. B) Time course of blood flow recovery (calculated as the ratio of blood flow in ischemic to contralateral foot; $n=5-6$ ). C) Representative micro-CT images showing the hindlimb vasculature in ND and DM mice undergoing HLI and administered with either vehicle, AG, or DAG for 14 days. The red oval indicates the measured area region of interest. D) Quantification of vascular volume by micro-CT analysis among all study groups $(n=5-6)$. E) Representative ischemic gastrocnemius sections stained with the endothelial marker isolectin B4 (green fluorescent) and a-smooth muscle actin (red fluorescent) to identify smooth muscle cells in the arterial wall (scale bar, $50 \mu \mathrm{m}$ ). F) Quantification of the capillary and (G) arteriole density (diameter $<50$ $\mu \mathrm{m})$ density in ischemic gastrocnemius of mice at 14 days post ischemia $(n=5-6)$. Data are presented as mean \pm S.E.M. Statistical comparisons were made using a RM two-way ANOVA, followed by Tukey's multiple comparisons post hoc tests. † Significantly different from ND Veh ( $\mathrm{P}<0.05$, †† $\mathrm{P}<0.01$, $<0.001)$; § Significantly different from ND DAG $(\S P<0.05$, §§§ $P<0.001)$; II Significantly different from DM Sham (I| || P< 0.01, || || || P< 0.001); \# Significantly different from DM Veh (\# $P<0.05$, \#\# P<0.01,

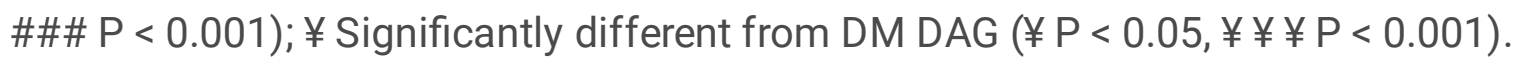



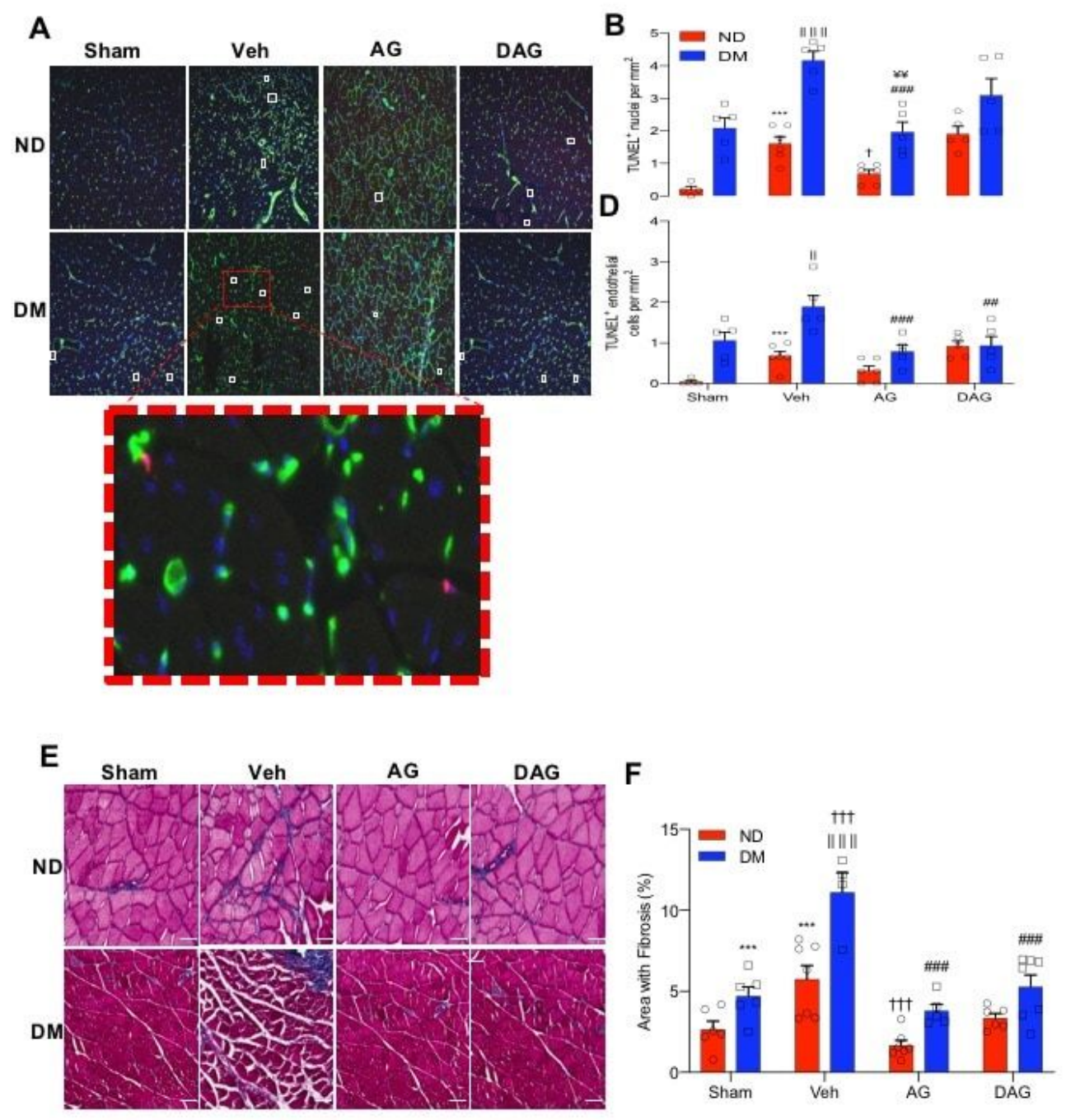

\section{Figure 9}

Exogenous ghrelin aids cell survival after hind limb ischemia in diabetic $\mathrm{db} / \mathrm{db}$ mice A) Representative images TUNEL-positive nuclei and (B) Quantification of TUNEL-positive nuclei $(n=4-6)$. C) Representative images TUNEL-positive endothelial cells in the gastrocnemius muscle 14 days post ischemia and (D) TUNEL-positive endothelial cells per mm2 $(n=4-6)$. Scale bars, $25 \mu \mathrm{m}$. E) Representative histological images of Masson's trichrome staining among all study groups following 14 days of HLI. F) 
Quantification of gastrocnemius muscle fibrosis $(n=4-7)$. Scale bar, $50 \mu \mathrm{m}$. Data are presented as mean \pm S.E.M. Statistical comparisons were made using a two-way ANOVA, followed by Tukey post hoc test (B, $D, F)$ * Significantly different from ND Sham (*** $P<0.001)$; † Significantly different from ND Veh $(† P<$ 0.05, t†† $P<0.001$ ); || Significantly different from DM Sham ( || $P<0.05$, || || || $P<0.001$ ); \# Significantly different from DM Veh (\#\# $P<0.01$, \#\#\# $P<0.001)$; $¥$ Significantly different from DM DAG $(¥ ¥ P<0.01)$.

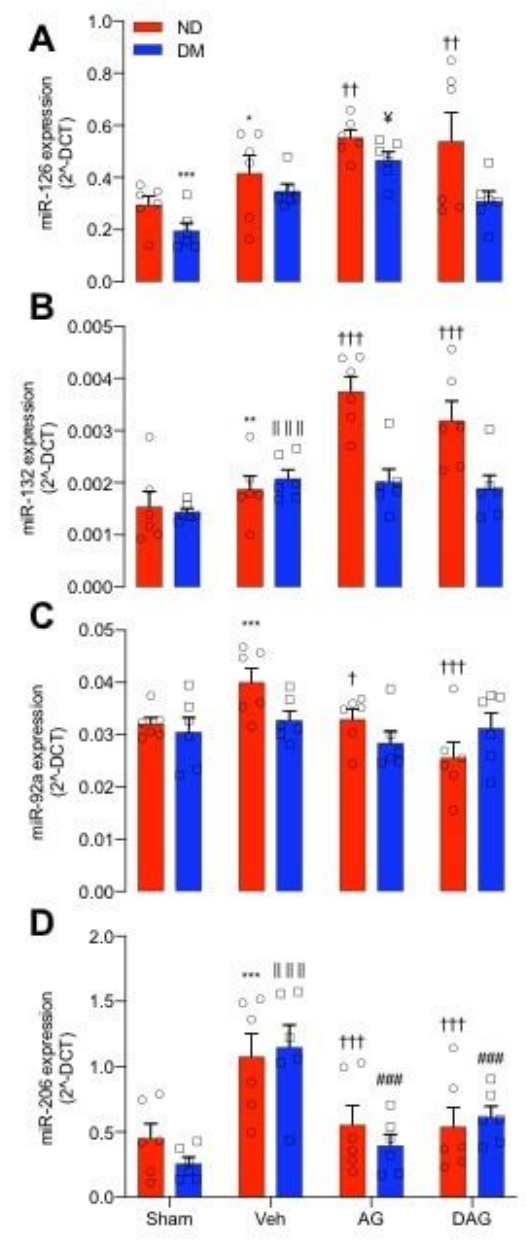

E
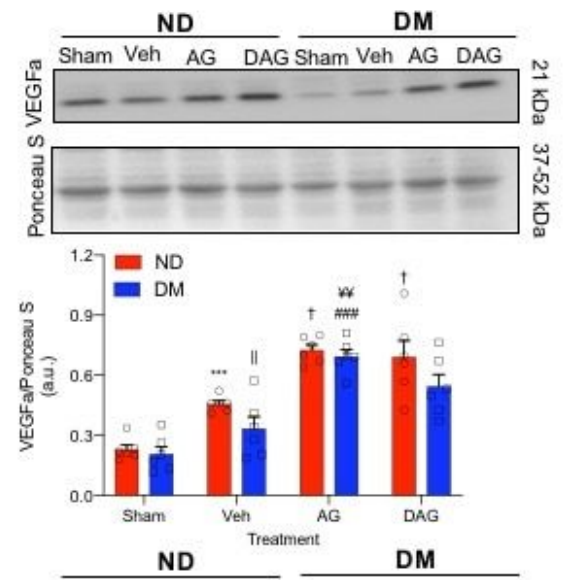

$\mathbf{F}$
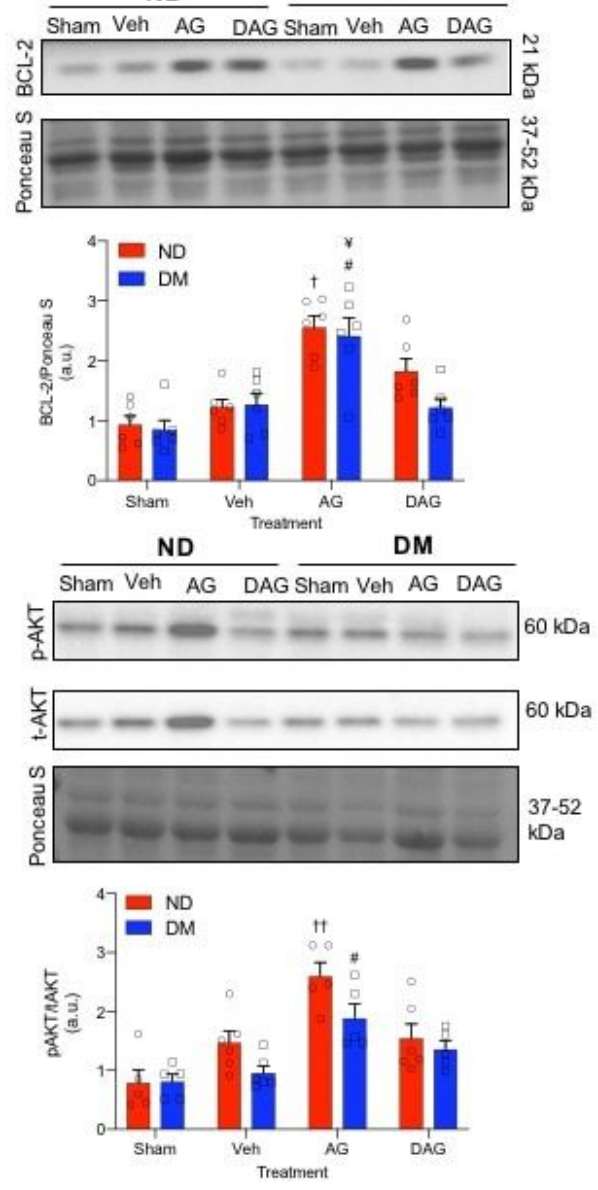

Figure 12 
Ghrelin treatment enhances pro-angiogenic and pro-survival miRNAs and proteins after hind limb ischemia in diabetic $\mathrm{db} / \mathrm{db}$ mice Bar graphs represent the average relative expression of $(A)$ miRNAs 126, (B) -132, (C) -92a, and (D) -206 normalised to the small nuclear U6 snRNA, which was used as a normaliser, among the study groups at 14 days following $\mathrm{HLI}(\mathrm{n}=5-6)$. Representative western blot images and protein quantification of (E) VEGFa, (F) BCL-2, and (G) pAKT in the ischemic gastrocnemius muscle among the study groups at 14 days following $\mathrm{HLI}(n=5-6)$. Ponceau-S staining was used as a loading control. Data are presented as mean \pm S.E.M. Statistical comparisons were made using two-way ANOVA, followed by Tukey post hoc test (A through $G)$. ${ }^{*}$ Significantly different from ND Sham $(* P<0.05$,

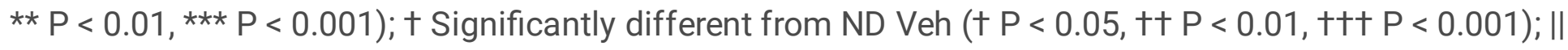
Significantly different from DM Sham (|| $P<0.05$, || || || $P<0.001)$; \# Significantly different from DM Veh (\# $P<0.05$, \#\#\# $P<0.001)$; $¥$ Significantly different from DM DAG $(¥ P<0.05$, ¥¥P<0.01) .

\section{Supplementary Files}

This is a list of supplementary files associated with this preprint. Click to download.

- SupplFig.8.tiff

- SupplFig.4.tiff

- SupplFig.3.tiff

- SupplementaryMaterialsandMethodsNEALE.docx

- SupplFig.1.tiff

- SupplFig.4.tiff

- SupplFig.3.tiff

- SupplFig.2.tiff

- SupplFig.1.tiff

- SupplementaryMaterialsandMethodsNEALE.docx

- SupplFig.7.tiff

- SupplFig.6.tiff

- SupplFig.5.tiff

- SupplFig.8.tiff

- SupplFig.7.tiff

- SupplFig.6.tiff

- SupplFig.5.tiff

- SupplFig.2.tiff 